\title{
The impact of exenatide (a GLP-1 agonist) on markers of inflammation and oxidative stress in normal human astrocytes subjected to various glycemic conditions
}

\author{
ŁUKASZ BUŁDAK, GRZEGORZ MACHNIK, ESTERA SKUDRZYK, \\ ALEKSANDRA BOŁDYS and BOGUSŁAW OKOPIEŃ \\ Department of Internal Medicine and Clinical Pharmacology, \\ School of Medicine in Katowice, Medical University of Silesia, 40-752 Katowice, Poland \\ Received September 6, 2018; Accepted January 3, 2019
}

DOI: $10.3892 /$ etm.2019.7245

\begin{abstract}
GLP-1 agonists such as exenatide and liraglutide are novel drugs for the treatment of diabetes and obesity. While improvements in glycemic control can rely on an incretin effect, the mechanisms behind the loss of weight following therapy have yet to be completely elucidated, and seem to be associated with alterations in eating habits, resulting from changes in cytokines e.g. interleukin $1 \beta$ (IL-1 $\beta$ ) and oxidative signaling in the central nervous system (CNS). Increased levels of IL-1 $\beta$ and reactive oxygen species have been demonstrated to exert anorexigenic properties, and astrocytes appear to actively participate in maintaining the integrity of the CNS, which includes the paracrine secretion of inflammatory cytokines and involvement in the redox status. Therefore, the present study decided to explore the influence of exenatide [a glucagon-like peptide 1 (GLP-1 agonist)] on inflammatory and oxidative stress markers in cultured human astrocytes as a potential target for weight reduction therapies. In an experimental setting, normal human astrocytes were subjected to various glycemic conditions, including $40 \mathrm{mg} / \mathrm{dl}$-hypoglycemic, $100 \mathrm{mg} / \mathrm{dl}$-normoglycemic and $400 \mathrm{mg} / \mathrm{dl}$-hyperglycemic, and exenatide, which is a GLP-1 agonist. The involvement of intracellular signaling by a protein kinase A (PKA) in the action of exenatide was estimated using a specific PKA inhibitor-PKI (14-22). The expression levels of IL-1 $\beta$, nuclear factor kappa $\kappa \mathrm{B}(\mathrm{NF} \kappa \mathrm{B})$, glial-fibrillary acidic protein (GFAP), p22 NADPH oxidase, glutathione peroxidase, catalase, superoxide dismutase 1 , and reactive oxidative species were measured. The present study demonstrated that
\end{abstract}

Correspondence to: Dr Łukasz Bułdak, Department of Internal Medicine and Clinical Pharmacology, School of Medicine in Katowice, Medical University of Silesia, 18 Medykow, 40-752 Katowice, Poland

E-mail: lbuldak@gmail.com

Key words: exenatide, oxidative stress, interleukin-1 $\beta$, Glucagon-like peptide-1, astrocytes varying glucose concentrations in the culture media did not affect the protein expression or the level of reactive oxygen species. Conversely, exenatide led to an increase in IL-1 $\beta$ in normoglycemic culture conditions, which was accompanied by the increased expression of p22, glutathione peroxidase and the reduced expression of GFAP. Changes in the expression of IL-1 $\beta$ and p22 were dependent on the activation of PKA. The present study concluded that exenatide predominantly affected astrocytes in normoglycemic conditions, and hypothesize that this impact demonstrated one of novel mechanisms associated with astrocyte signaling that may contribute to weight loss.

\section{Introduction}

Diabetes and obesity are reaching pandemic scale worldwide (1). The targeted and effective methods for the pharmacological treatment of obesity are sparse, but there have been several new therapies introduced into clinical practice to treat type 2 diabetes mellitus [e.g. glucagon-like peptide 1 (GLP-1) agonists, dipeptidyl peptidase-4 (DPP-4) inhibitors or sodium/glucose cotransporter 2 (SGLT-2) inhibitors] which may ease weight reduction (SGLT-2 inhibitors, GLP-1 agonists). Weight loss is crucial to diabetic subjects (2) and it is rather easy to explain the mechanism during the therapy with drugs that induce glycosuria (leading to a glucose loss of up to $300 \mathrm{kcal}$ per day with urine). However, it is not that clear for the other group of novel antidiabetic drugs.

GLP-1 is a hormone that is produced in the gastrointestinal (GI) tract and is liberated after the ingestion of meals, leading to a myriad of effects (i.e. the incretin effect). It lowers GI motility, increases insulin secretion, reduces glucagon level and reduces food intake (3). As a result, a tendency to reduce the body weight of subjects with type 2 diabetes during the treatment with GLP-1 agonists was noted (4). This observation led to exploratory studies, which showed the weight-reducing potency of those drugs in non-diabetic subjects (5). Subsequently, liraglutide (a GLP-1 agonist) has been approved for the treatment of obesity in subjects without diabetes (6).

Mechanisms behind the weight loss seen during the therapy with GLP-1 agonists are multifactorial and not well understood (7). Nevertheless, it seems that the impact on the central 
nervous system and eating habits are crucial. There are several sites in the central nervous system (e.g. hypothalamus) that are linked with eating habits (8). Neurons that form those centers have limited access to GLP-1 agonists due to the existence of the blood brain barrier (BBB). The BBB is formed from several different cell types, including astrocytes. Astrocytes not only protect neurons, but also actively participate in the transduction of signals from the bloodstream to neurons $(9,10)$, which suggest that they have paracrine modulating capabilities in neuronal signaling. A significant positive correlation between astrogliosis, represented by an increased expression of glial-fibrillary acidic protein (GFAP), and obesity in animal studies have been noted (11). The mediators of astrocyte signaling may include inflammatory pathways (IL-1 $\beta$ and $\mathrm{NF} \kappa \mathrm{B}$ ) and oxidative stress (reactive oxygen species-ROS; the expression of proteins associated with oxidative stress-p22 NADPH oxidase; and anti-oxidative enzymes-catalase, superoxide dismutase and glutathione peroxidase) (12). It is thought that inflammatory cytokines and excessive oxidative stress may have anorexigenic properties $(13,14)$. Inflammation and oxidative stress are exaggerated by variations in glucose concentrations, especially during prolonged hyperglycemia in diabetes (15). Recent studies have shown that astrocytes are actively involved in the inflammatory and oxidative processes associated with the pathogenesis of several diseases (e.g. major depressive disorder, epilepsy) (16). In addition, a growing body of evidence suggests their key participation in the pathogenesis of obesity and diabetes $(17,18)$. Brain magnetic resonance imaging studies in human subjects showed a significant correlation between glial activation and obesity (19). Finally, astrocytes are now considered to be contributors in the CNS signaling system (20).

Several studies have explored the impact of GLP-1 agonists on astrocytes, but they have been performed on animal behavioral models $(21,22)$ or animal-derived astrocytes in an in vitro setting (23). We have noted that there are few data on the impact of GLP-1 agonists on human non-malignant astrocytes.

Therefore, we conceived a study to assess the short-term impact of exenatide (a GLP-1 agonist) on IL-1 $\beta$, NFאB, GFAP and redox status in normal human astrocytes (NHA) cultured in vitro. Due to the clinical use of GLP-1 agonists in the treatment of type 2 diabetes and obesity without coexisting diabetes we decided to perform a set of experiments in various glycemic conditions ranging from hypoglycemia $(40 \mathrm{mg} / \mathrm{dl})$, via normoglycemia (100 mg/dl) to hyperglycemia $(400 \mathrm{mg} / \mathrm{dl})$. In order to explore whether the changes were dependent on PKA activation, which is one of the major downstream pathways responsible for the cellular effects (e.g. inflammatory and oxidative) of GLP-1 agonists $(24,25)$, we performed experiments using a selective PKA inhibitor [PKI (14-22)].

\section{Materials and methods}

Cell culture. Normal human astrocytes (NHA) cell line was purchased from Lonza, cat. no. CC-2565 (Cell lab, Warsaw, Poland). Cells were thawed accordingly to the supplier's recommendations and cultivated in the astrocyte growth medium (AGM), which consisted of astrocyte basal medium (cat. no. CC-3187) supplemented with AGM SingleQuots (cat. no. CC-4123), both obtained from Lonza. All experiments were done within 7th cell passages. The day before each experiment, culture medium was replaced with glucose-containing medium at predefined concentrations (40, 100 and $400 \mathrm{mg} / \mathrm{dl})$. On the day of the experiment, exenatide, which belongs to GLP-1 agonists (Exendin-4, cat. no. E7144, Sigma-Aldrich; Merck KGaA, Poznań, Poland), was reconstituted in water accordingly to the manufacturer's recommendations. Afterwards it was diluted in the culture medium to achieve $1 \mathrm{mM}$ solution of exenatide. In selected culture dishes, $30 \mathrm{~min}$ prior to the addition of exenatide, PKI (14-22) (a pharmacological inhibitor of protein kinase A) was added to the medium at the final concentration of $10 \mu \mathrm{M}$. NHA were incubated at $37^{\circ} \mathrm{C}$ in the atmosphere containing $95 \%$ of air and $5 \%$ of $\mathrm{CO}_{2}$ in a $\mathrm{CO}_{2}$ incubator (Hera-Cell, Thermo Fischer Scientific, Inc., Grand Island, NY, USA). Those culture conditions are referred further as 'standard culture conditions'. Experiments were performed for $24 \mathrm{~h}$. Afterwards samples were collected and stored as described below. HeLa cell line was a gift from the Department of Biotechnology and Genetic Engineering, Medical University of Silesia, Sosnowiec, Poland. HeLa cells were cultivated in the Dulbecco's modified Eagle's medium (DMEM) supplemented with $10 \%$ of bovine fetal serum and $50 \mu \mathrm{g} / \mathrm{ml}$ of gentamycin (cat. no. P04-01550, Pan-Biotech, $\mathrm{GmbH}$, Immuniq, Żory, Poland). HeLa cells were used as a reference cell line that shows low expression of GLP-1 receptors (26).

Resazurin assay. In order to estimate the viability of cultured cells in the experimental setting the resazurin assay was used. The choice of the assay was based on its reliability, simplicity and sensitivity in the assessment of cell viability (27). Briefly, one day before the experiment, $5 \times 10^{4}$ NHA were seeded on 24-well culture plate (SPL Life Sciences Co., Ltd., Immuniq) in $0,5 \mathrm{ml}$ of AGM growth medium. On the next day, $50 \mu 1$ of resazurin dye solution was added into each well (cat. no. TOX-8, Sigma-Aldrich; Merck KGaA). Afterwards cells were incubated for $2 \mathrm{~h}$ under standard culture conditions and shaked gently by using rotation platform set at $100 \mathrm{rpm}$. The decrease in absorbance at a wavelength of $600 \mathrm{~nm}$ was measured spectrophotometrically using xMark Microplate Absorbance Spectrophotometer (Bio-Rad Laboratories, Inc., Warsaw, Poland). Additional measurements were done at a reference wavelength of $690 \mathrm{~nm}$ and were subtracted from the previous that were obtained at $600 \mathrm{~nm}$.

ROS assessment. As shown by other researchers, a spectrometry-based assay for determining intracellular ROS production and its level at the final phase of experiments was performed using Nitrotetrazolium Blue chloride (NBT) dye (No. cat. N6876, Sigma-Aldrich; Merck KGaA) (28). A stock solution (1\% NBT) was added into cell culture to the final concentration of $0,1 \%$. Cells were then incubated for $24 \mathrm{~h}$ under standard conditions in 24 -well plates $\left(5 \times 10^{4}\right)$. Thereafter, supernatant was completely removed and $200 \mu \mathrm{l}$ of PBS solution was added into the cells. A sonication procedure at $80 \%$ power intensity for $10 \mathrm{sec}$. was done in order to disrupt the cells and liberate NBT into the solution. Finally, absorbance at the wavelength of $550 \mathrm{~nm}$ was measured using xMark Microplate Absorbance Spectrophotometer (Bio-Rad Laboratories, Inc.). 
Western blot analysis. In the western blot analysis, human-specific antibodies were used as follows: For GLP-1 receptor (GLP-1R): Anti-GLP-1R, (Bioss Antibodies Inc. Woburn, MA, USA); for nuclear factor $\kappa \mathrm{B}(\mathrm{NF}-\kappa \mathrm{B}$ p65): Anti-NF- $\kappa$ B (Cell Signaling Technology, Lab-JOT Ltd., Warsaw, Poland); for NADPH oxidase (p22): Anti-CYBA; for GFAP: GFAP Polyclonal Antibody (Thermo Fischer Scientific, Inc., Warsaw, Poland); for glutathione peroxidase (GPx): Anti-GPX1; for catalase (Cat): Anti-Catalase and for superoxide dismutase-1 (SOD1): Anti-SOD1 (all from Sigma-Aldrich; Merck KGaA). For quantitative analysis, a reference glyceraldehyde 3-phosphate dehydrogenase (GAPDH) protein, $\beta$-actin or lamin B1 were estimated in every sample using an anti-GAPDH antibody, Actin-beta antibody (both obtained from Thermo Fischer Scientific, Inc.) or Anti-Lamin B1 antibody (Abcam, Symbiosis, Rotmanka, Poland).

Cells were cultured on 12-well culture plates $\left(1 \times 10^{5}\right.$ cells per well) (SPL Life Sciences Co., Ltd.). Prior to cell lysis, plates were placed on ice and cells were washed briefly with $500 \mu \mathrm{l}$ of ice-cold PBS. Protein extraction was done using $150 \mu \mathrm{l}$ of cold RIPA buffer supplemented with $1.5 \mu \mathrm{l}$ of Halt Protease Inhibition Cocktail (1:100 v/v) per well (both chemicals from Thermo Fischer Scientific, Inc.). The amount of total protein was measured in each sample by bicinchoninic acid assay (BCA assay) technique and total protein concentration was calculated according to the standard curve based on bovine serum albumin (BSA) solutions of known protein concentration (Thermo Fischer Scientific, Inc.). Proteins from cell lysates were separated by means of electrophoresis in polyacrylamide gel in the presence of ColorPlus Prestained Protein Marker (New England Biolabs, Lab-Jot, Warsaw, Poland). $20 \mu \mathrm{g}$ of total protein was loaded into gel slots. After separation, proteins were right away electroblotted onto PVDF membrane (Merck Millipore, Poznań, Poland). Membranes were blocked by incubation in $3 \%$ bovine serum albumin (BSA) solution in Tris-buffered saline (1X TBS) for two hours and then membranes were placed in 3\% BSA/1X TTBS (TBS supplemented with $0,05 \%$ of Tween-20) containing one type of antibody at a final dilution of 1:1,000, except for anti-GAPDH antibody that was diluted to a greater extent $(1: 2,000)$. Incubations were performed for $1 \mathrm{~h}$ at ambient temperature with continuous rocking. Then, after two washes in TTBS for 10-min each, an Anti-rabbit IgG (whole molecule)-peroxidase antibody (No. cat. A0545, Sigma-Aldrich; Merck KGaA) was added (antibody dilution: 1:10,000 in 3\% BSA/TTBS). Incubation was performed for one hour under continuous rocking. Finally, after three washes (2X TTBS for 5 min. each and $1 \mathrm{X}$ TBS for $5 \mathrm{~min}$.), a specific chemiluminescent signal was developed (Pierce ECL Western Blotting Substrate, Thermo Fischer Scientific, Inc.). After development, membranes were digitalized using ChemiDoc-It Imaging System (Analytik Jena, Jena, Germany). Measurements of integrated optical density representing the amount of the protein of interest in a sample were done using ImageJ software.

ELISA. Cell supernatants were sampled from cell culture directly before cell lysis for protein analysis was performed (1x10 $0^{5}$ cells per well). After removing from cell culture plate, supernatants were aliquoted in $500 \mu \mathrm{l}$ and frozen at $-80^{\circ} \mathrm{C}$ for

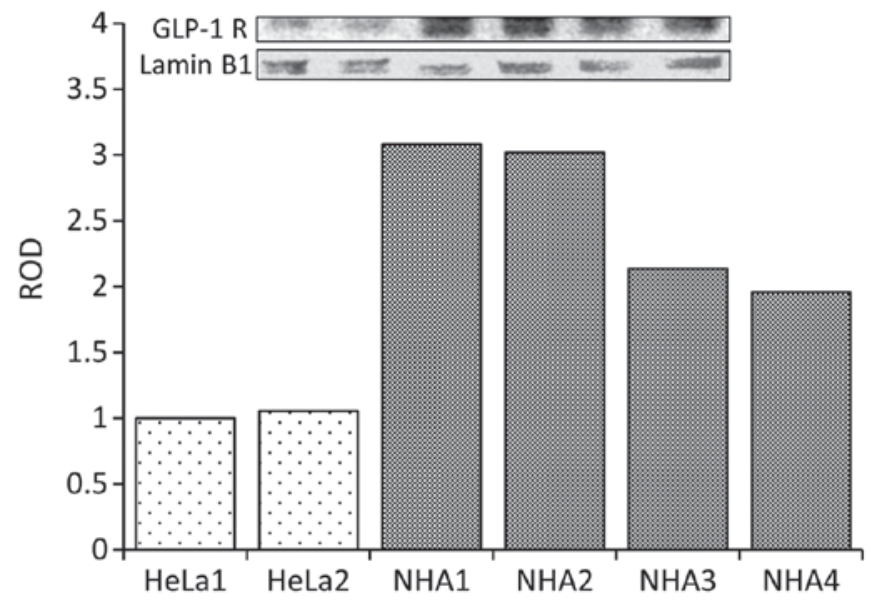

Figure 1. Compared to HeLa (human cervical carcinoma cell line) NHA show abundant expression of GLP-1 receptors. HeLa1 and HeLa2-two separate cultures of HeLa cells. NHA1-NHA4-four separate cultures of normal human astrocytes. ROD-relative optical density of western blot bands expressed in comparison to HeLa1.

ELISA analysis. The concentration of interleukin-1 $\beta$ in NHA cell supernatant was estimated using Human IL-1 $\beta$ ELISA kit (Diaclone, cat. no. 850.006, Immuniq)-according to the supplier's instructions for use. The level of IL-1 $\beta$ showed the level of expression and secretion of this cytokine to the culture media. Every sample was analyzed in triplicate. The measurements of absorbance at the wavelength of $450 \mathrm{~nm}$ were done using xMark Microplate Absorbance Spectrophotometer (Bio-Rad Laboratories, Inc.). A second measurement at a reference wavelength of $620 \mathrm{~nm}$ was done and this value was subtracted from that of $450 \mathrm{~nm}$.

Statistical analysis. The normality of distribution of data was evaluated using Shapiro-Wilk's test. Afterwards data were analyzed using one-way ANOVA test with post-hoc Tukey test and were reported as mean $\pm \mathrm{SE}$. The $\mathrm{P}$ level below 0.05 was considered as statistically significant.

\section{Results}

The expression of GLP-1R. The first objective of the study was to examine the presence of potential targets of the therapy by confirming the expression of GLP-1 receptors in NHA. The experiments showed that these cells expressed substantial amount of GLP-1 receptors (Fig. 1).

The viability of NHA in culture conditions. In the next step of the study, the viability of cells was assessed in all selected glycemic conditions and in the absence or presence of exenatide in culture media. We estimated that the viability of NHA in all culture conditions ranged between 98.76 and $108.7 \%$. No statistically significant differences between treatment groups were observed. Therefore, data were not presented in the figure.

The impact of various glycemic conditions and exenatide on the level of interleukin $1 \beta(I L-1 \beta)$ in the culture medium. In the next step of the experiment, the impact of selected glycemic conditions and exenatide on a marker of inflammation (IL-1 $\beta$ ) 

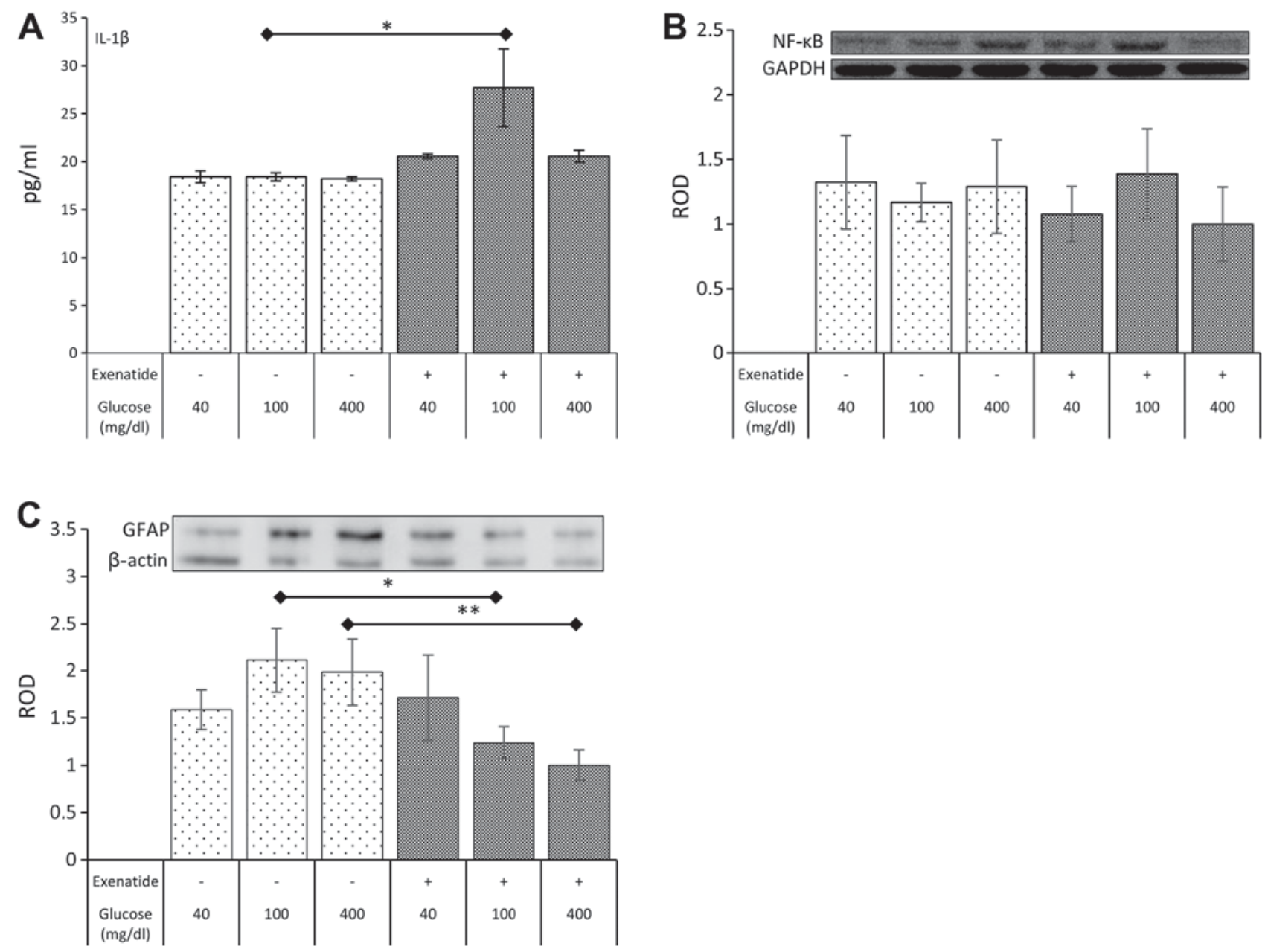

Figure 2. The impact of various glycemic conditions and exenatide on the concentration of IL-1 $\beta$ secreted to culture medium by NHA (A) and the level of expression of NFkB (B) and GFAP (C) in NHA. Data expressed as mean \pm SE. Asterisk indicates level of statistical significance: ${ }^{*} \mathrm{P}<0.05,{ }^{* *} \mathrm{P}<0.01$.

was estimated. The IL-1 $\beta$ level was not altered in any of the selected glycemic conditions without exenatide. However, exenatide led to a rise $(51 \% ; \mathrm{P}=0.022)$ in the concentration of IL-1 $\beta$ in normoglycemic cultures (Fig. 2A). The impact of the GLP-1 agonist in hypo- and hyperglycemia was statistically insignificant.

The impact of various glycemic conditions and exenatide on the expression of nuclear factor kappa $B(N F \kappa B)$. Despite the impact of exenatide on IL-1 $\beta$ levels the expression of NF $\kappa B$ remained unaffected in all experimental conditions (Fig. 2B).

The impact of various glycemic conditions and exenatide on the expression of GFAP. The GFAP expression was not affected by different glycemic culture conditions. However, a significant reduction in the expression of GFAP was noted in cells exposed to exenatide under normoglycemic (42\%; $\mathrm{P}=0.014)$ and hyperglycemic $(56 \% ; \mathrm{P}=0.005)$ conditions. Exenatide failed to impact the expression of GFAP in hypoglycemic conditions (Fig. 2C).

The impact of various glycemic conditions and exenatide on the expression of NADPH oxidase (p22). In the next step of our study, a set of experiments estimating the oxidative stress was performed. The first results (without a GLP-1 agonist) showed a positive trend toward higher expression of p22 that was concurrent with the increasing concentration of glucose in the culture medium, but it was not statistically significant. On the other hand, exenatide showed a strong influence on the p22 expression. GLP-1 agonist nearly doubled the expression of p22 in hypoglycemic conditions (94\% increase in p22 expression; $\mathrm{P}=0.001)$, while the impact on $\mathrm{p} 22$ in normoglycemia resulted in an increase, reaching $47 \%(\mathrm{P}=0.021)$. No impact of exenatide was noted in hyperglycemic culture conditions (Fig. 3A). Of note, the expression of p22 in astrocytes in the presence of exenatide decreased with increasing concentrations of glucose. The magnitude of the reduction between hyperglycemia and hypoglycemia reached $36 \%(\mathrm{P}=0.011)$.

The impact of various glycemic conditions and exenatide on the expression of glutathione peroxidase (GPx). Several other experiments focused on the expression of proteins connected with the protection against oxidative stress. Glutathione peroxidase expression was not affected by changes in the glucose concentration without exenatide. However the GLP-1 agonist was able to significantly elevate the level of GPx (76\% increase; $\mathrm{P}=0.015$ ) in NHA cultured in normoglycemic conditions. However, the GPx expression after exposure to exenatide in hyperglycemia and hypoglycemia remained unaffected (Fig. 3B). 

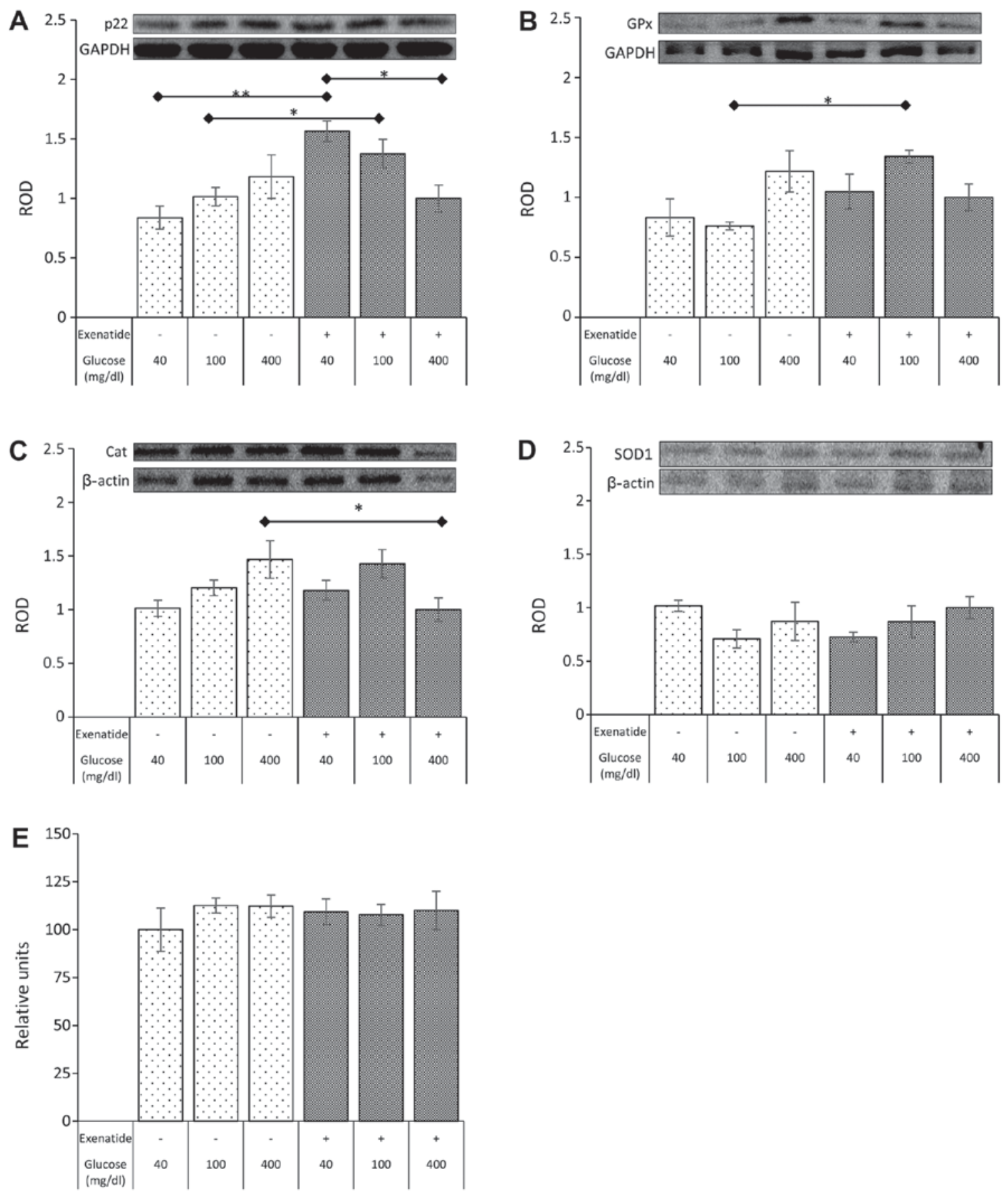

Figure 3. The impact of various glycemic conditions and exenatide on the level of expression of p22 (A), GPx (B), Cat (C), SOD1 (D) and ROS level (E) in NHA. Data expressed as mean \pm SE. Asterisks indicate level of statistical significance: ${ }^{*} \mathrm{P}<0.05 ;{ }^{* *} \mathrm{P}<0.01$.

The impact of various glycemic conditions and exenatide on the expression of catalase (Cat). Regardless of the concentration of glucose in culture media, the expression of catalase, the second most important antioxidative enzyme, did not change. However, exenatide led to a surprising decrease $(42 \%$; $\mathrm{P}=0.022)$ in catalase expression in NHA cultured under hyperglycemic conditions (Fig. 3C).

The impact of various glycemic conditions and exenatide on the expression of superoxide dismutase 1 (SOD1). Experiments on the expression of SOD1 did not show any statistically significant impact of various glycemic conditions, regardless of the presence or absence of exenatide (Fig. 3D).
The impact of various glycemic conditions and exenatide on the level of reactive oxygen species (ROS). Noteworthy, despite changes in the expression of p22 and antioxidative enzymes, experiments did not show any significant influence of culture conditions on the amount of ROS generated by NHA (Fig. 3E).

Experiments with protein kinase A inhibition. The activation of protein kinase A is the main mechanism of intracellular downstream signaling from GLP-1 receptors. Therefore, in order to explore whether alterations in concentration of IL-1 $\beta$ and the expression of $\mathrm{p} 22$, catalase and glutathione peroxidase were the result of GLP-1 activation, a set of 

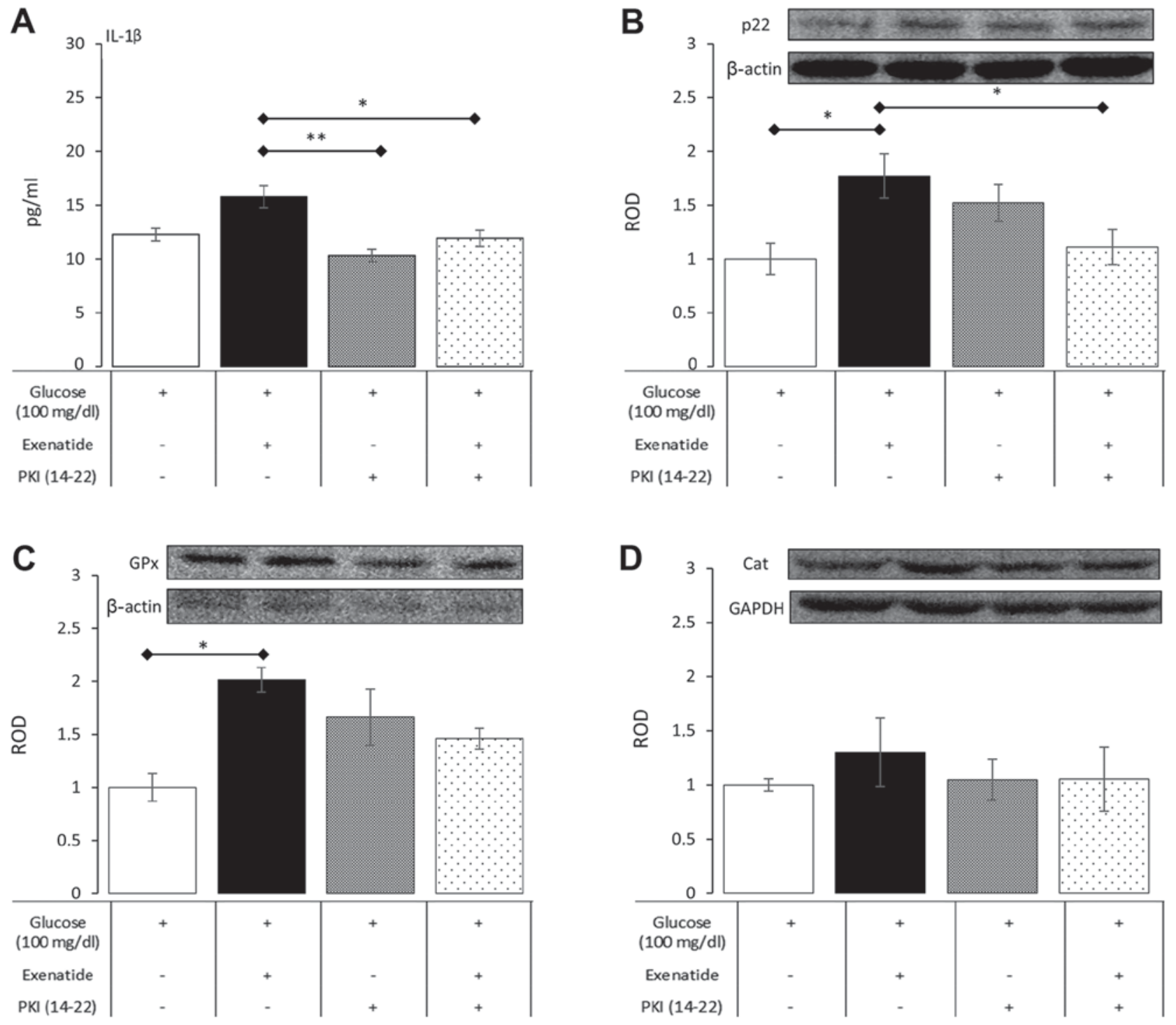

Figure 4. The impact of PKI (14-22)-a selective PKA inhibitor-on the concentration of IL-1 $\beta$ (A) and the expression of p22 (B), GPx (C) and Cat (D) in NHA subjected to exenatide in normoglycemic conditions. Data expressed as mean $\pm \mathrm{SE}$. Asterisk indicates level of statistical significance: ${ }^{*} \mathrm{P}<0.05 ;{ }^{* *} \mathrm{P}<0.01$.

experiments with PKI (14-22) (a pharmacological inhibitor of protein kinase A) was performed under normoglycemic conditions. PKI (14-22), as a sole addition to the culture medium, did not affect the measured variables compared to control cultures, which were supplemented only with glucose at a final concentration of $100 \mathrm{mg} / \mathrm{dl}$. However, a significant dampening of the influence of exenatide was noted when PKI (14-22) was added to culture media (Fig. 4). In detail, PKI (14-22) significantly reduced IL-1 $\beta$ concentration by $24 \%(\mathrm{P}=0.047)$ in exenatide-treated NHA, resulting in levels comparable to those seen in control cultures. PKI (14-22) was also able to inhibit p22 expression by $37 \%(\mathrm{P}=0.048)$ in cells treated with PKI (14-22) plus exenatide compared to cells treated with exenatide alone. The impact of PKI (14-22) on the effects of exenatide on GPx and catalase showed only a trend between PKA activation and their expression, but this did not reach statistical significance. These experiments showed that the impact of exenatide on NHA relies to some extent on the activation of protein kinase $\mathrm{A}$, which is a major signaling transduction pathway from GLP-1 receptors.

\section{Discussion}

Our study was focused on the assessment of inflammatory status and redox potential of NHA subjected to hypo-, normo- and hyperglycemia in the absence or presence of exenatide (a GLP-1 agonist). According to our results, short-term exposure only to varying glycemic conditions was not connected with changes in the studied parameters of the NHA. Noteworthy, we noted increased inflammatory activity (expressed by an elevated level of IL-1 $\beta$ in culture media) in cells subjected to the treatment with exenatide in normoglycemic conditions, which was accompanied by the reduced expression of GFAP in normoglycemic and hyperglycemic conditions. We also observed that exenatide affected redox status in NHA. There was an increase in the expression of p22 in NHA cells treated with exenatide in hypoglycemic and normoglycemic conditions. These changes were accompanied by the increased expression of the antioxidative enzyme GPx under normoglycemic conditions and a decrease in Cat expression in cells subjected to both hyperglycemia and exenatide. 
Finally, we confirmed that the observed effects were partially reversible by a pharmacological inhibitor of PKA (PKI 14-22) in normoglycemic cultures.

The CNS inflammatory signaling has recently been connected with the pathogenesis of obesity (29). For several years, it has been known that astrocytes actively participate in the process of signal modulation and transduction in the CNS. They gather inputs from the bloodstream, transport particles through the BBB and convey their signaling by the secretion of cytokines and changing local redox status (30). According to our results, varying the glucose concentration in the culture media did not change the level of IL-1 $\beta$ secreted and did not affect NFKB expression. However, we noted a remarkable increase in IL-1 $\beta$ level in normoglycemic cultures treated with exenatide We believe that the increased level of IL-1 $\beta$ might be a way to convey signals in the CNS by astrocytes. The increased expression of IL- $1 \beta$ was accompanied by a reduced glial activation in normoglycemic and hyperglycemic condition, which was shown by a reduction in the GFAP expression. Of note, we did not see a significant change in the expression of NFKB under similar conditions. This observation seems to be in contrast to previously published experiments with GLP-1 agonists in human macrophages. Human macrophages exposed to exenatide expressed less IL-1 $\beta$ (31). However, the NFкB binding activity remained unaffected in exenatide-treated macrophages (32). GLP-1 agonist reduced IL-1 $\beta$ expression and inflammatory cytokine levels even more extensively in macrophages that were concurrently subjected to lipopolysaccharide (LPS). Similar results were observed in LPS-treated astrocytes, showing a significant reduction in IL-1 $\beta$ levels in cells treated with LPS (33). Moreover, in mice receiving intrahippocampal LPS injection, a significant reduction in $\mathrm{NF} \times \mathrm{B}$ and IL-1 $\beta$ was reported in the presence of exenatide (21). However, others published data showing that the GLP-1 agonist without strong proinflammatory stimuli actually increased the expression of proinflammatory cytokines (34). Therefore, it seems that the discrepancy may be a result of a different cellular response in severe inflammation (e.g. infection) and in physiological conditions (which should be reflected in culture conditions that is devoid of strong inflammatory stimuli). In our study, which was conceived without strong proinflammatory stimulus, we showed that exenatide increased the expression of IL-1 $\beta$, which, in paracrine way, may be responsible for altered feeding behaviors. Previously, it was shown that IL-1 $\beta$ had anorexigenic properties and could affect body weight at the hypothalamic level (35). Of note, we noted that exenatide had the ability to elevate the level of IL-1 $\beta$ under normoglycemic culture conditions, which is concordant with the extended therapeutic indications of GLP-1 agonists to treat obesity even without coexisting diabetes. Remarkably, in contrast to the findings in animal-derived astrocytes, we did not observe a concurrent rise in $\mathrm{NF} \kappa \mathrm{B}$ expression with IL-1 $\beta$ (36). However, there are several differences between the experimental settings. Astrocytes in animal studies were purified cells derived from mice brain samples. The culture consisted of $>95 \%$ astrocytes, but the remaining population might contain highly reactive microglial cells that significantly altered IL-1 expression due to their great ability to amplify IL-1 $\beta$ expression on external stimuli (37). Furthermore, in the study by Wang et al (36), it was established that NFאB activation and subsequent IL- $1 \beta$ expression was dependent on the coincident aggravated oxidative stress. In our experiments on NHA, we did not observe any significant changes in the ROS level, which may have prevented the activation of $\mathrm{NF} \kappa \mathrm{B}$. The possible explanation for an increase in IL-1 $\beta$ by exenatide is the impact of the GLP-1 agonist on mitogen-associated protein kinases (MAPKs) (38). MAPKs are downstream kinases that are activated by PKA; in our experiments, we showed that PKA inhibitor was able to reduce the IL-1 $\beta$ levels in the culture media to levels comparable to those of control control. Finally, it seems that not only interspecies differences may lead to unexpected results. It was shown that the inflammatory responsiveness of cells derived from patients with diabetes and healthy volunteers differed significantly (39). IL-1 $\beta$ was very low in peripheral blood mononuclear cells (PBMCs) that were derived from healthy population and their response to GLP-1 receptor stimulation was negligible; however, in cells from patients with diabetes (hyperglycemic), the basal IL-1 $\beta$ level was high and strongly reduced by GLP-1 agonists. This may be a peculiar type of a legacy effect that results in varied responses to stimuli in different background conditions. The issue of different response of NHA to exenatide in our experiments is still not completely clear, but the strength of our experimental setting and the use of commercially available human non-malignant cell line should reduce potential confounders. We speculate that the direct impact of exenatide on NHA IL-1 $\beta$ signaling may be at least partly responsible for the mechanisms behind weight reduction during GLP-1-based therapies, even without coexisting diabetes. Furthermore we showed reduced astrogliosis, which seems to be connected with beneficial effects in the treatment of diet-induced obesity in animal models (40).

Diabetes and obesity are connected with increased systemic and local oxidative stress (15). More importantly, endoplasmic reticulum (ER) stress in astrocytes has been connected to several diseases, including obesity (41). At the cellular level, ER stress led to the increased generation of ROS in mitochondria. Astrocytes may play a dual role in the pathogenesis of obesity and diabetes. These cells may protect neurons from hostile environment (42) or may become a source and transducer of detrimental stimuli (43). Our results showed that the short-term exposure of astrocytes to varying glucose concentrations in culture media was not sufficient to change the ROS level. On the other hand, exenatide in hypoglycemic and normoglycemic conditions increased the expression of $\mathrm{p} 22$, which is a major source of ROS. This change in the expression of p22 might lead to a local increase in ROS generation. However, it seems that exenatide action on astrocytes is multifactorial, while it simultaneously affected the expression of anti-oxidative enzymes (GPX in normoglycemic cultures and catalase in hyperglycemic conditions). As a result of the above-mentioned changes in protein expression, there have been no changes in the level of ROS. These results might suggest that exenatide, in normoglycemic conditions, affected the redox status by altering the anti-oxidative capacity, expressed by the elevated GPx level. This resulted in an unchanged level of ROS, despite a significant increase in the expression of major ROS donor. ROS are considered sensitizers of behavior, which may lead to over-nutrition (44). Recent studies show that 
exenatide may have neuroprotective properties in an in vivo model of neurodegenerative diabetic rats by a reduction of oxidative stress (45). Excessive oxidative stress was also abolished by exenatide in the ischemic model of cardiac muscle injury (46). Noteworthy, it was also noted that excessive oxidative stress that occurred in rat model of cerebral stroke led to a significant reduction in the level of GLP-1 receptors (47). This loss might be at least partly compensated by the exogenously administered exenatide. Those results show a significant impact of exenatide on markers of oxidative stress in animal or in vitro models of various acute injuries. On the other hand, we performed a study that was deprived of strong inflammatory (e.g. LPS, TNF alpha) or oxidative stressors (e.g. $\mathrm{H}_{2} \mathrm{O}_{2}$ ). This was intentional, as such experimental conditions may more accurately reflect naturally occurring changes in the microenvironment. Our previous experiments on human macrophages also failed to show a spectacular impact of exenatide on ROS levels, while a parallel minute increase in p22 expression was noted (48). Only after strong pro-inflammatory stimulation was a clear protective effect of GLP-1 agonism against oxidative stress noted. In the current experiment, we did not wish to expose astrocytes to supraphysiological stimuli and also chose the NHA cell line instead of animal or human neoplastic lines, which may be less or more responsive than other cell lines. However, we consider that these experimental conditions should more closely reflect naturally occurring processes. While the lack of impact on the global ROS level in our experimental setting may be surprising, it should be kept in mind that local changes in redox status resulting from the altered expression of NADPH oxidase and antioxidative enzymes may also take part in cell signaling.

In summary, we have found that astrocytes were susceptible to exenatide stimulation and that the net effect seemed to rely on background glucose concentration. Exenatide significantly increased the secretion of IL-1 $\beta$ in normoglycemic conditions and reduced GFAP expression in normo- and hyperglycemic cultures. The expression of p22 NADPH oxidase was also elevated in normoglycemic and hypoglycemic conditions. Those alterations were accompanied by changes in the expression of antioxidative enzymes (GPx and Cat) under normoglycemic and hyperglycemic conditions. The current findings showed some novel aspects of paracrine signaling in normal human astrocytes exposed to exenatide in selected glycemic condition. While it is tempting to attribute them as being responsible for the impact on eating habits and reduced food intake, further studies in different experimental conditions (e.g. co-culture, in vivo settings) are warranted to explore such an assumption.

The limitation of our study must also be taken into account. The in vitro setting definitely does not reflect the complexity of cellular interplay in the CNS and entire organism. The relatively short-term culture in contrast to the long-lasting burden of hyper- or hypoglycemia may have a slightly different impact on astrocytes. Therefore further testing in a more complex setting, as above-mentioned co-cultures, are vital to attempt the transition of cellular into clinical research. However, we believe that the use of human astrocytes and the non-inflammatory culture conditions contribute substantially to the currently available data.

\section{Acknowledgements}

Authors express their gratitude to Mrs. Jaroslawa Sprada (Department of Pharmacology, School of Medicine in Katowice, Medical University of Silesia, Katowice, Poland) for her expert technical support.

\section{Funding}

The study was supported by research grant from Medical University of Silesia (grant no. KNW-1-095/N/8/0).

\section{Availability of data and materials}

The datasets used and/or analyzed during the current study are available from the corresponding author on reasonable request.

\section{Authors' contributions}

$Ł B$ conceived the study, performed data analysis, and wrote the manuscript. GM conducted the in vitro cultures and laboratory assays and reviewed the manuscript. ES conducted the in vitro cultures and laboratory assays and reviewed manuscript. $\mathrm{AB}$ analyzed data and reviewed manuscript. BO overviewed the experiments and was responsible for the interpretation of data, and critically revising the manuscript for important intellectual content. All authors read and approved the final manuscript.

\section{Ethics approval and consent to participate}

Not applicable.

\section{Patient consent for publication}

Not applicable.

\section{Competing interests}

The authors declare that they have no competing interests.

\section{References}

1. Le Magueresse-Battistoni B, Labaronne E, Vidal H and Naville D: Endocrine disrupting chemicals in mixture and obesity, diabetes and related metabolic disorders. World J Biol Chem 8: 108-119, 2017.

2. Kaul S: Mitigating cardiovascular risk in type 2 diabetes with antidiabetes drugs: A review of principal cardiovascular outcome results of EMPA-REG OUTCOME, LEADER, and SUSTAIN-6 trials. Diabetes Care 40: 821-831, 2017.

3. Andersen A, Lund A, Knop FK and Vilsbøll T: Glucagon-like peptide 1 in health and disease. Nat Rev Endocrinol 14: 390-403, 2018.

4. Santilli F, Simeone PG, Guagnano MT, Leo M, Maccarone MT Di Castelnuovo A, Sborgia C, Bonadonna RC, Angelucci E, Federico V, et al: Effects of liraglutide on weight loss, fat distribution, and $\beta$-cell function in obese subjects with prediabetes or early type 2 diabetes. Diabetes Care 40: 1556-1564, 2017.

5. Astrup A, Carraro R, Finer N, Harper A, Kunesova M, Lean ME, Niskanen L, Rasmussen MF, Rissanen A, Rössner S, et al: Safety, tolerability and sustained weight loss over 2 years with the once-daily human GLP-1 analog, liraglutide. Int J Obes (Lond) 36: 843-854, 2012.

6. Nuffer WA and Trujillo JM: Liraglutide: A new option for the treatment of obesity. Pharmacotherapy 35: 926-934, 2015. 
7. Martin-Jiménez CA, Gaitán-Vaca DM, Echeverria V, González J and Barreto GE: Relationship between obesity, Alzheimer's disease, and Parkinson's disease: An astrocentric view. Mol Neurobiol 54: 7096-7115, 2017.

8. Grill HJ and Hayes MR: The nucleus tractus solitarius: A portal for visceral afferent signal processing, energy status assessment and integration of their combined effects on food intake. Int $\mathrm{J}$ Obes (Lond) 33 (Suppl 1): S11-S15, 2009.

9. Croft W, Dobson KL and Bellamy TC: Plasticity of neuron-glial transmission: Equipping glia for long-term integration of network activity. Neural Plast 2015: 765792, 2015.

10. Zhao Z, Nelson AR, Betsholtz C and Zlokovic BV: Establishment and dysfunction of the blood-brain barrier. Cell 163: 1064-1078, 2015.

11. Buckman LB, Thompson MM, Moreno HN and Ellacott KL: Regional astrogliosis in the mouse hypothalamus in response to obesity. J Comp Neurol 521: 1322-1333, 2013.

12. Moretti R, Pansiot J, Bettati D, Strazielle N, Ghersi-Egea JF, Damante G, Fleiss B, Titomanlio L and Gressens P: Blood-brain barrier dysfunction in disorders of the developing brain. Front Neurosci 9: 40, 2015.

13. de Git KC and Adan RH: Leptin resistance in diet-induced obesity: The role of hypothalamic inflammation. Obes Rev 16: 207-224, 2015.

14. García MC, Wernstedt I, Berndtsson A, Enge M, Bell M, Hultgren O, Horn M, Ahrén B, Enerback S, Ohlsson C, et al: Mature-onset obesity in interleukin-1 receptor I knockout mice. Diabetes 55: 1205-1213, 2006.

15. Boyer F, Vidot JB, Dubourg AG, Rondeau P, Essop MF and Bourdon E: Oxidative stress and adipocyte biology: Focus on the role of AGEs. Oxid Med Cell Longev 2015: 534873, 2015.

16. Dossi E, Vasile F and Rouach N: Human astrocytes in the diseased brain. Brain Res Bull 136: 139-156, 2018.

17. Chowen JA, Argente-Arizón P, Freire-Regatillo A, Frago LM, Horvath TL and Argente J: The role of astrocytes in the hypothalamic response and adaptation to metabolic signals. Prog Neurobiol 144: 68-87, 2016

18. Chowen JA, Argente J and Horvath TL: Uncovering novel roles of nonneuronal cells in body weight homeostasis and obesity. Endocrinology 154: 3001-3007, 2013.

19. Thaler JP, Yi CX, Schur EA, Guyenet SJ, Hwang BH, Dietrich MO, Zhao X, Sarruf DA, Izgur V, Maravilla KR, et al: Obesity is associated with hypothalamic injury in rodents and humans. J Clin Invest 122: 153-162, 2012.

20. Papouin T, Dunphy J, Tolman M, Foley JC and Haydon PG: Astrocytic control of synaptic function. Philos Trans R Soc Lond B Biol Sci 372: pii: 20160154, 2017

21. Huang HJ, Chen YH, Liang KC, Jheng YS, Jhao JJ, Su MT, Lee-Chen GJ and Hsieh-Li HM: Exendin-4 protected against cognitive dysfunction in hyperglycemic mice receiving an intrahippocampal lipopolysaccharide injection. PLoS One 7: e39656, 2012.

22. Reiner DJ, Mietlicki-Baase EG, McGrath LE, Zimmer DJ, Bence KK, Sousa GL, Konanur VR, Krawczyk J, Burk DH Kanoski SE, et al: Astrocytes regulate GLP-1 receptor-mediated effects on energy balance. J Neurosci 36: 3531-3540, 2016.

23. Gullo F, Ceriani M, D'Aloia A, Wanke E, Constanti A, Costa B and LecchiM:Plantpolyphenols and exendin-4 preventhyperactivityand TNF- $\alpha$ release in LPS-treated in vitro neuron/astrocyte/microglial networks. Front Neurosci 11: 500, 2017.

24. Bao Y, Jiang L, Chen H, Zou J, Liu Z and Shi Y: The neuroprotective effect of liraglutide is mediated by glucagon-like peptide 1 receptor-mediated activation of cAMP/PKA/CREB pathway. Cell Physiol Biochem 36: 2366-2378, 2015.

25. Lee YS and Jun HS: Anti-inflammatory effects of GLP-1-based therapies beyond glucose control. Mediators Inflamm 2016 3094642, 2016.

26. Vinet L, Lamprianou S, Babič A, Lange N, Thorel F, Herrera PL, Montet X and Meda P: Targeting GLP-1 receptors for repeated magnetic resonance imaging differentiates graded losses of pancreatic beta cells in mice. Diabetologia 58: 304-312, 2015.

27. Czekanska EM: Assessment of cell proliferation with resazurin-based fluorescent dye. Methods Mol Biol 740: 27-32, 2011

28. Nitti M, Furfaro AL, Traverso N, Odetti P, Storace D, Cottalasso D, Pronzato MA, Marinari UM and Domenicotti C: PKC delta and NADPH oxidase in AGE-induced neuronal death. Neurosci Lett 416: 261-265, 2007.

29. Douglass JD, Dorfman MD and Thaler JP: Glia: Silent partners in energy homeostasis and obesity pathogenesis. Diabetologia 60: 226-236, 2017
30. Etchegoyen M, Nobile MH, Baez F, Posesorski B, González J, Lago N, Milei J and Otero-Losada M: Metabolic syndrome and neuroprotection. Front Neurosci 12: 196, 2018.

31. Bułdak Ł, Machnik G, Bułdak RJ, Łabuzek K, Bołdys A, Belowski D, Basiak M and Okopień B: Exenatide (a GLP-1 agonist) expresses anti-inflammatory properties in cultured human monocytes/macrophages in a protein kinase $\mathrm{A}$ and $\mathrm{B} / \mathrm{Akt}$ manner. Pharmacol Rep 68: 329-337, 2016.

32. Bułdak Ł, Machnik G, Bułdak RJ, Łabuzek K, Bołdys A and Okopień B: Exenatide and metformin express their anti-inflammatory effects on human monocytes/macrophages by the attenuation of MAPKs and NFKB signaling. Naunyn Schmiedebergs Arch Pharmacol 389: 1103-1115, 2016.

33. Iwai T, Ito S, Tanimitsu K, Udagawa $\mathrm{S}$ and Oka J: Glucagon-like peptide-1 inhibits LPS-induced IL-1beta production in cultured rat astrocytes. Neurosci Res 55: 352-360, 2006.

34. Shirazi R, Palsdottir V, Collander J, Anesten F, Vogel H, Langlet F, Jaschke A, Schürmann A, Prévot V, Shao R, et al: Glucagon-like peptide 1 receptor induced suppression of food intake, and body weight is mediated by central IL-1 and IL- 6 . Proc Natl Acad Sci USA 110: 16199-16204, 2013.

35. Schéle E, Benrick A, Grahnemo L, Egecioglu E, Anesten F, Pálsdóttir V and Jansson JO: Inter-relation between interleukin (IL)-1, IL-6 and body fat regulating circuits of the hypothalamic arcuate nucleus. J Neuroendocrinol 25: 580-589, 2013.

36. Wang J, Li G, Wang Z, Zhang X, Yao L, Wang F, Liu S, Yin J, Ling EA, Wang L and Hao A: High glucose-induced expression of inflammatory cytokines and reactive oxygen species in cultured astrocytes. Neuroscience 202: 58-68, 2012.

37. Liu Y, Biarnés Costa M and Gerhardinger C: IL-1 $\beta$ is upregulated in the diabetic retina and retinal vessels: cell-specific effect of high glucose and IL-1 $\beta$ autostimulation. PLoS One 7: e36949, 2012.

38. Varga ZV, Giricz Z, Liaudet L, Haskó G, Ferdinandy P and Pacher P: Interplay of oxidative, nitrosative/nitrative stress, inflammation, cell death and autophagy in diabetic cardiomyopathy. Biochim Biophys Acta 1852: 232-242, 2015.

39. He L, Wong CK, Cheung KK, Yau HC, Fu A, Zhao HL, Leung KM, Kong AP, Wong GW, Chan PK, et al: Anti-inflammatory effects of exendin-4, a glucagon-like peptide-1 analog, on human peripheral lymphocytes in patients with type 2 diabetes. J Diabetes Investig 4: 382-392, 2013.

40. Nerurkar PV, Johns LM, Buesa LM, Kipyakwai G, Volper E, Sato R, Shah P, Feher D, Williams PG and Nerurkar VR: Momordica charantia (bitter melon) attenuates high-fat diet-associated oxidative stress and neuroinflammation. J Neuroinflammation 8: 64, 2011.

41. Martin-Jiménez CA, García-Vega Á, Cabezas R, Aliev G, Echeverria V, González J and Barreto GE: Astrocytes and endoplasmic reticulum stress: A bridge between obesity and neurodegenerative diseases. Prog Neurobiol 158: 45-68, 2017.

42. Tanaka J, Toku K, Zhang B, Ishihara K, Sakanaka M and Maeda N: Astrocytes prevent neuronal death induced by reactive oxygen and nitrogen species. Glia 28: 85-96, 1999.

43. Maragakis NJ and Rothstein JD: Mechanisms of disease: Astrocytes in neurodegenerative disease. Nat Clin Pract Neurol 2: 679-689, 2006.

44. Jang EY, Ryu YH, Lee BH, Chang SC, Yeo MJ, Kim SH, Folsom RJ, Schilaty ND, Kim KJ, Yang CH, et al: Involvement of reactive oxygen species in cocaine-taking behaviors in rats. Addict Biol 20: 663-675, 2015.

45. Abdelwahed OM, Tork OM, Gamal El Din MM, Rashed L and Zickri M: Effect of glucagon-like peptide-1 analogue; Exendin-4, on cognitive functions in type 2 diabetes mellitus; possible modulation of brain derived neurotrophic factor and brain Visfatin. Brain Res Bull 139: 67-80, 2018.

46. Chang G, Liu J, Qin S, Jiang Y, Zhang P, Yu H, Lu K, Zhang N, Cao L, Wang Y, et al: Cardioprotection by exenatide: A novel mechanism via improving mitochondrial function involving the GLP-1 receptor/cAMP/PKA pathway. Int J Mol Med 41: 1693-1703, 2018

47. Kim S, Jeong J, Jung HS, Kim B, Kim YE, Lim DS, Kim SD and Song YS: Anti-inflammatory effect of glucagon like peptide-1 receptor agonist, exendin-4, through modulation of IB1/JIP1 expression and JNK signaling in stroke. Exp Neurobiol 26: 227-239, 2017

48. Bułdak Ł, Łabuzek K, Bułdak RJ, Machnik G, Bołdys A and Okopień B: Exenatide (a GLP-1 agonist) improves the antioxidative potential of in vitro cultured human monocytes/macrophages. Naunyn Schmiedebergs Arch Pharmacol 388: 905-919, 2015 